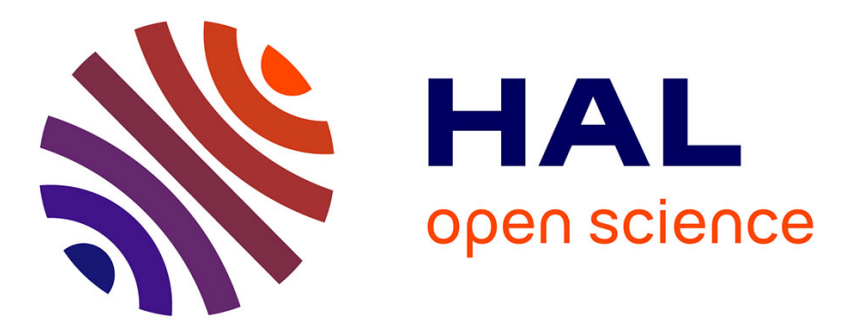

\title{
L'Union européenne face aux normes internationales de reporting financier (IFRS) : une mise en perspective historique
}

Didier Bensadon

\section{- To cite this version:}

Didier Bensadon. L'Union européenne face aux normes internationales de reporting financier (IFRS): une mise en perspective historique. Politiques et Management public, 2016, 33 (2), 10.3166/pmp.33.2.135-154 . hal-02284378

\section{HAL Id: hal-02284378 \\ https://hal.science/hal-02284378}

Submitted on 11 Sep 2019

HAL is a multi-disciplinary open access archive for the deposit and dissemination of scientific research documents, whether they are published or not. The documents may come from teaching and research institutions in France or abroad, or from public or private research centers.
L'archive ouverte pluridisciplinaire HAL, est destinée au dépôt et à la diffusion de documents scientifiques de niveau recherche, publiés ou non, émanant des établissements d'enseignement et de recherche français ou étrangers, des laboratoires publics ou privés. 


\section{L'Union européenne face aux normes internationales de reporting financier (IFRS) : une mise en perspective historique}

\section{> Didier Bensadon}

Université Paris-Dauphine, PSL Research University, CNRS, [UMR 7088], DRM, MOST, 75016 Paris, France

\section{Résumé}

Pendant près de vingt-cinq ans l'harmonisation comptable européenne s'est concrétisée par l'adoption de directives. Au milieu des années 1990, la Commission européenne annonçait qu'elle abandonnait ce mode d'harmonisation comptable pour les sociétés cotées publiant des comptes consolidés afin d'opter pour un processus de normalisation comptable internationale sous l'égide de I'IASC de l'époque. De nombreux travaux ont déjà été publiés sur les raisons de ce changement de stratégie. Dans cette recherche, nous souhaitons comprendre les raisons pour lesquelles les critiques de la Commission européenne à l'encontre des directives ont été si violentes. À partir des archives disponibles autour de la négociation de la Septième directive et en nous appuyant sur les travaux de Diggle et Nobes (1994) et de ceux de Van Hulle et Van der Tas (1995) nous mettons en lumière les stigmates laissées par la négociation de la Septième directive européenne, la remise en cause l'efficacité des options inhérentes aux directives et l'influence de l'Organisation Internationale des Commissions de Valeurs favorisant un rapprochement vers avec l'organisme de normalisation comptable international. Cet article contribue ainsi à une meilleure connaissance de l'évolution des modes de régulation comptable.

(c) 2016 IDMP/Lavoisier SAS. Tous droits réservés Mots clés : harmonisation comptable européenne, normes IFRS, normalisation comptable, consolidation des comptes, Septième directive européenne, histoire de la comptabilité.

\section{Abstract}

The European Union facing International Financial Reporting Standards (IFRS): a historical perspective. For nearly twenty-five years the European accounting harmonization is reflected in the adoption of directives. In the mid 1990s, the European Commission announced that it was abandoning this accounting harmonization mode for listed companies publishing consolidated accounts. It opted for a more international 
accounting standardization process under the aegis of the International Accounting Standards Committee. The reasons for this change in strategy have already been analyzed in several studies. The present research aims to understand why the criticism of the European Commission against the directives were so violent. From the archives available around the negotiation of the Seventh Directive and relying on the work of Diggle and Nobes (1994) and those of Van Hulle and Van der Tas (1995) we highlight the scars left by negotiation the Seventh Council Directive, questioning the effectiveness of the inherent options directives and the influence of the International Organization of Securities Commissions to promote reconciliation with the International Accounting Standards Committee. This article contributes to a better understanding of changes in accounting regulation.

(c) 2016 IDMP/Lavoisier SAS. Tous droits réservés

Keywords: Accounting regulation, group accounting, accounting history, seventh European directive,

Accounting harmonization, IFRS.

\section{Introduction}

Depuis le $1^{\text {er }}$ janvier 2005 les sociétés cotées sur un marché financier réglementé ont l'obligation d'utiliser les normes comptables internationales (IFRS) pour publier leurs comptes consolidés. Ces normes, mises au point par l'International Accounting Standards Board, s'inspirent d'un cadre conceptuel qui est le fruit d'une histoire économique, financière et sociale issue du monde anglo-saxon. Même s'il existe un processus de convergence des règles comptables françaises vers les standards internationaux, des divergences fondamentales subsistent. À la question de savoir : à quoi sert la comptabilité ? Certains considèrent qu'elle doit uniquement servir les intérêts des investisseurs et qu'elle doit informer sur le potentiel économique d'une firme (modèle anglo-saxon). Pour d'autres, la comptabilité sert à évaluer un patrimoine avec, en point d'orgue, la préparation d'un bilan qui rassemble en un seul document les droits et les obligations d'une entité dotée de la personnalité morale (modèle continental). Aujourd'hui, pour la préparation et la publication des comptes sociaux, ces deux visions de la comptabilité sont entremêlées. En revanche, pour l'élaboration des comptes consolidés, c'est une approche purement économique et financière qui est privilégiée où le principe substance over form constitue un élément central.

À la question de savoir : qui doit participer au processus de normalisation? Les régulateurs nationaux européens sont partagés. Certains pays comme la France, l'Allemagne, la Belgique ou encore l'Italie considèrent que c'est la collégialité qui doit primer dans le processus d'élaboration des normes comptables. L'idée étant d'aboutir à une norme comptable qui puisse satisfaire les attentes de toutes les parties prenantes impliquées dans la création de valeur de l'entreprise. En France, les différentes structures mises en place au sortir de la Seconde guerre mondiale ont favorisé la concertation et le consensus des parties impliquées au sein du Conseil national de la comptabilité puis, de l'Autorité des normes comptables (Azan, 2002). D'autres pays tels que le Royaume-Uni, l'Irlande, le Danemark ou encore les Pays-Bas considèrent que la normalisation comptable doit relever exclusivement de la compétence de la profession comptable. 
La question qui nous intéresse ici porte sur le mode de régulation comptable au niveau européen. Il s'agit d'un point fondamental au cœur des enjeux politiques et idéologiques autour de la comptabilité (Colasse (2005), Frison-Roche (2011), Richard et al. (2014). Au milieu des années 1990, sur cette question de la régulation comptable, la Commission européenne a opéré un tournant radical dans le processus d'élaboration des normes comptables destinées à être utilisées pour l'établissement et la publication des comptes de consolidés des sociétés faisant appel public à l'épargne. Elle s'est en effet engagée dans une voie visant à déléguer à l'IASC (devenu IASB depuis) la préparation des normes comptables utilisables dans l'Union européenne.

Cette décision de la Commission européenne a déjà été largement commentée. La valeur juridique des processus de normalisation comptable et leur inscription dans les transformations de la régulation juridique ont été questionnées par Raybaud-Turrillo (2001). De son côté, Chiapello (2005) souligne que les nouvelles normes transforment la conception de l'entreprise. Elle explicite également le faisceau de facteurs qui a abouti à cette transformation institutionnelle majeure pour le capitalisme européen. Pour sa part, Colasse (2005) revient sur le processus institutionnel ayant conduit la Commission européenne à prendre cette décision. Chiapello et Medjad (2007) mettent en évidence que le transfert de compétences au secteur privé va bien au-delà des formes habituelles de délégation. Ils montrent que si cet effacement de la puissance publique a des causes variées, celles-ci doivent être principalement recherchées au sein de l'Union Européenne. Enfin, Chambost et Klee (2009) proposent d'approfondir cette question sous l'angle de l'articulation de l'expertise et du politique, telle que conceptualisée par Habermas, en la situant au niveau supranational de l'Union européenne.

L'objectif de cet article n'est pas de revenir sur les jeux d'acteurs qui ont permis de faire en sorte que la Commission européenne opte pour une telle solution. Les activités de lobbying des grandes entreprises internationales ou des cabinets d'audit au sein des organismes de normalisation comptable sont connues (Sutton, 1984; Walton, 2003; Colasse, 2004).

Cet article cherche plutôt à comprendre pourquoi la Commission européenne a émis des critiques si virulentes à l'égard des directives européennes lorsqu'elle a annoncé son changement de stratégie en matière d'harmonisation comptable.

Cette question se pose avec d'autant plus d'acuité que la France a transposé récemment ${ }^{1}$ la directive comptable unique (2013/34/UE) qui abroge les Quatrième et Septième directives européennes.

Cette recherche s'inscrit dans une perspective interprétative (Hoarau, 1995). Elle porte notamment sur les interactions entre l'évolution de l'élaboration de la norme et les changements de l'environnement social. C'est la méthode historique qui est ici privilégiée car nous considérons avec Cailluet et Lemarchand (2013) et Zimnovitch (2013) que la mise en lumière du passé permet de mieux appréhender le présent; en l'occurrence, le choix de la Commission européenne en matière d'harmonisation comptable. Cette recherche s'appuie sur des archives privées et publiques. Les premières sont constituées des notes person-

\footnotetext{
${ }^{1}$ La directive comptable unique (2013/34/UE) a été transposée le 23 juillet 2015 par l'ordonnance $\mathrm{n}^{\circ}$ 2015-900 du 23 juillet 2015 relative aux obligations comptables des commerçants, le décret $\mathrm{n}^{\circ} 2015$ 903 du 23 juillet 2015 relatif aux obligations comptables des commerçants et l'arrêté du 4 décembre 2015 portant homologation des règlements n² 2015-6 et n²015-7 du 23 novembre 2015 l'Autorité des normes comptables.
} 
nelles de Bertrand d'Illiers ${ }^{2}$ alors responsable des affaires comptables de la Commission des Opérations de Bourse (COB). Il fut l'un des trois représentants français lors de la discussion de la Septième directive européenne à Bruxelles durant les années 1970. Les secondes sont des archives publiques (comptes rendus des débats au sein des différentes instances communautaires).

La première section de cet article est consacrée à une mise en perspective des fondements juridiques des directives européennes dans le champ de l'harmonisation comptable européenne (1.1). En ce qui concerne la régulation comptable, Colasse (2009) distingue deux niveaux : l'harmonisation et la normalisation. L'harmonisation est censée autoriser une diversité des pratiques comptables et vise seulement à établir des équivalences entre elles. Elle est en principe moins contraignante que la normalisation. La normalisation a quant à elle pour objet l'application de normes identiques dans le même espace géographique. Une présentation succincte des trois directives comptables permet de constater les réalisations en matière d'harmonisation comptable européenne (1.2). Les critiques virulentes que formule la Commission européenne à l'encontre des directives sont mises en évidence et analysées (1.3). Ces critiques sont publiées dans un document officiel ${ }^{3}$ de la Commission où elle annonce son changement de stratégie en matière d'harmonisation comptable.

La seconde section apporte des éléments de réponse qui permettent de mieux comprendre les critiques formulées à l'égard des directives européennes. Diggle et Nobes (1994), Van Hulle et Van der Tas (1995) ou en encore Vangermeersch (1985) nous ont déjà livré des analyses fouillées sur la gestation laborieuse de la Septième directive et notamment sur le glissement progressif d'un texte d'inspiration de droit comptable allemand, vers un texte plus en phase avec les pratiques anglo-saxonnes. Par conséquent, notre ambition n'est pas de retracer une nouvelle fois l'histoire de la difficile élaboration de la Septième directive européenne, mais d'utiliser cette histoire pour éclairer la décision de la Commission européenne. Il est néanmoins nécessaire de présenter succinctement les travaux préliminaires du Groupe d'études des experts-comptables européens (2.1) et les avis du Conseil économique et Social et du Parlement (2.2) avant d'insister sur la phase de dénouement autour des travaux du Coreper (2.3). C'est au niveau de cette institution que les oppositions des différentes délégations européennes sont les plus virulentes et où il est possible de comprendre la difficulté à obtenir un accord politique. Cet épisode autour des travaux Coreper s'appuie sur des archives totalement inédites : celles du négociateur français Bertrand d'Illiers.

La troisième section propose des éléments de discussion visant à apporter un éclairage sur les motivations de la Commission européenne à abandonner toute velléité d'harmonisation comptable européenne pour les sociétés devant présenter des comptes consolidés. La

\footnotetext{
${ }^{2}$ L'auteur remercie M. Bertrand d'Illiers (ancien responsable des affaires comptables de la COB) représentant français à Bruxelles pour la négociation de la Septième directive européenne pour lui avoir confié ses archives professionnelles privées sur la préparation de la Septième directive. Ces dernières se composent essentiellement : de notes de la $\mathrm{COB}$, de notes du secrétariat général du comité interministériel pour les questions de coopération économique européenne, des rapports du Conseil des communautés européennes, des comptes rendus sommaires du Comité des représentants permanent à Bruxelles (COREPER), des résumés des travaux du groupe des questions économiques (établissements et services), des notes de la Présidence du Secrétariat du Conseil (DGC III) et des nombreuses notes manuscrites prises par Bertrand d'Illiers.

${ }^{3}$ Commission européenne : Com 95(358) et Com 00(59)
} 
contribution de cet article est de mettre en évidence quatre facteurs explicatifs : la lenteur du processus d'harmonisation par directives (3.1), la multiplicité des options qui nuit à l'objectif de comparabilité des états financiers consolidés (3.2), un niveau de pratique très hétérogène de la consolidation des comptes entre les États membres (3.3) et la montée en puissance de l'IASC sous les auspices de l'OICV (3.4).

\section{Grandeur et décadence de la directive européenne comme dispositif d'harmoni- sation comptable des comptes consolidés}

Dès la fin des années 1960, plusieurs chantiers visant à coordonner les législations nationales en matière de droit des sociétés sont lancés. La directive européenne est alors retenue comme base juridique de cette harmonisation. Plusieurs directives intéressant le champ de la comptabilité furent adoptées durant les années 1980. Au milieu des années 1990, la Commission européenne change radicalement de stratégie et adresse des critiques virulentes au processus d'harmonisation comptable via les directives. Elle annonce, dès 1995, sa volonté d'abandonner ce dispositif d'harmonisation au profit d'un rapprochement avec l'IASB.

\subsection{Une base juridique : la directive européenne}

Le traité de Rome a prescrit l'établissement d'un programme général pour la suppression des restrictions à la liberté d'établissement qui existent à l'intérieur de la communauté. Dans son article 54.3-g, figure une liste non exhaustive des matières pour lesquelles le Conseil et la Commission sont autorisés à élaborer des réglementations communautaires. C'est au sein même de cette liste, que se trouve la base juridique de la coordination des législations nationales en matière de droit des sociétés. Depuis 1968, le Conseil a arrêté toute une série de mesures communautaires portant coordination du droit des sociétés au sein de l'Union européenne ${ }^{4}$. Toutes ces mesures présentent des caractéristiques communes qui concernent : les principes directeurs, l'étendue de la coordination, la nature des actes juridiques communautaires utilisés et les sociétés concernées (Reynaud-Fourton, 1984).

L'existence de la directive en tant que norme juridique communautaire est prévue à l'article 189 du Traité de Rome : «la directive lie tout État membre destinataire quant au résultat à atteindre, tout en laissant aux instances nationales la compétence quant à la forme et aux moyens ». La directive présente deux caractéristiques majeures. D'une part, elle n'a d'effet obligatoire qu'à l'égard des États membres qu'elle désigne. D'autre part, elle

\footnotetext{
${ }^{4}$ Cette coordination a été réalisée par l'adoption de directives : la Première (1968) établissant des règles communautaires en matière de publicité de certains documents et informations, de validité des engagements de la société et de la nullité de la société; la Deuxième (1976) sur la constitution de la société anonyme, le maintien de l'intégrité du capital de la société et l'augmentation ou la réduction de son capital; la Troisième (1978) sur l'établissement de règles communautaires en matière de fusion; la Quatrième (1978) qui traite de la structure et du contenu des comptes annuels des sociétés individuelles, des méthodes d'évaluation, de la publicité et du contrôle; la Sixième (1982) qui concerne la coordination des règles sur la scission des sociétés anonymes relevant du droit d'un État membre; la Septième (1983) sur la coordination des législations nationales sur les comptes consolidés; la Huitième (1984) concernant l'agrément des personnes chargées du contrôle légal des documents comptables.
} 
permet un compromis entre la reconnaissance de pouvoirs aux institutions communautaires et le maintien de certaines compétences au niveau des États.

Le contenu des directives se limite à fixer un objectif général. En revanche, le choix des moyens et de la forme nécessaires pour atteindre le résultat escompté sont laissés à l'appréciation souveraine des États membres.

\subsection{Les avancées en matière d'harmonisation comptable européenne (1978-1985)}

L'harmonisation comptable européenne a conduit à mettre au point et à adopter trois directives importantes dans le domaine comptable. La Quatrième directive adoptée en 1978 a été transposée dans la loi du 30 avril 1983. Cette directive visait à harmoniser la présentation des états financiers dans les États membres. Son adoption a été l'occasion d'introduire le concept d'image fidèle jusque-là inconnu dans les référentiels comptables continentaux.

La seconde réalisation significative est l'adoption, en juin 1983, de la Septième directive européenne sur les comptes consolidés. Elle a été transposée dans le droit interne par la loi du 3 janvier 1985. Cette directive visait à fournir un cadre harmonisé pour la préparation et la publication des comptes consolidés pour les sociétés dont le siège social est situé dans un État membre de la Communauté Économique Européenne de l'époque.

Ces deux directives sont aujourd'hui abrogées. La directive comptable unique 2013/34/ UE du 23 juin 2013, a remplacé les deux directives comptables européennes. Elle a été transposée dans le droit national le 23 juillet 2015.

Publiée au Journal Officiel de la Communauté Européenne le 12 mai 1984, la huitième directive européenne est consacrée aux professionnels chargés du contrôle légal des documents comptables. Cette directive définit les conditions d'honorabilité, les compétences théoriques et pratiques requises des professionnels chargés de l'audit légal.

Les apports de ces directives ne sont pas négligeables. La qualité des rapports annuels des sociétés de l'Union européenne s'est considérablement améliorée depuis l'adoption des directives (Colasse, 2009). Les sociétés ayant leur siège social dans les différents États membres ont fait converger leurs pratiques. Toutefois, des différences existent quant au traitement de certaines opérations (opérations en devises, impôts différés, crédit-bail). Comme le montre Colasse (2009), la directive n'aborde que les points sur lesquels un consensus a été possible entre les différents représentants des États membres. Les points délicats sur lesquels il n'y a pas de consensus possible sont écartés du champ de la directive. Par ailleurs, l'élaboration d'une directive est un processus très long et sa mise en application dans les États membres l'est davantage encore. Comme le montre le tableau $\mathrm{n}^{\circ} 1$, les délais d'introduction des directives européennes dans les législations nationales des États membres sont très variables.

Ils aboutissent finalement à obérer l'objectif d'harmonisation des pratiques comptables. Ce problème a été identifié par la Commission qui pour y remédier a institué un comité de contact en charge de la conseiller sur des compléments ou des amendements à apporter aux directives. Face à un manque de résultats probants, ce comité fut lui-même remplacé en 1990 par le forum consultatif de la comptabilité, sans davantage de succès cependant (Klee, Chambost et Boisselier, 2012).

Sous la pression de l'OICV et face au développement de la globalisation financière et de la financiarisation des économies, la Commission européenne pose clairement la question de la pertinence d'une l'harmonisation comptable strictement européenne. 
Tableau 1 : Années de transposition des directives dans les droits nationaux des États membres

\begin{tabular}{|l|c|c|c|}
\hline & $\begin{array}{c}\text { Date d'entrée } \\
\text { dans la CEE }\end{array}$ & $\begin{array}{c}\text { Quatrième directive } \\
\text { Adoptée en 1978 }\end{array}$ & $\begin{array}{c}\text { Septième directive } \\
\text { Adoptée en 1983 }\end{array}$ \\
\hline France & 1958 & 1983 & 1985 \\
\hline Pays-Bas & 1958 & 1983 & 1988 \\
\hline Luxembourg & 1958 & 1984 & 1988 \\
\hline Belgique & 1958 & 1985 & 1990 \\
\hline Allemagne & 1958 & 1985 & 1985 \\
\hline Italie & 1958 & 1991 & 1991 \\
\hline Danemark & 1973 & 1981 & 1990 \\
\hline Royaume-Uni & 1973 & 1981 & 1989 \\
\hline Irlande & 1973 & 1986 & 1992 \\
\hline Grèce & 1981 & 1986 & 1987 \\
\hline
\end{tabular}

Tiré de Colasse (2009)

\section{3. les critiques de la Commission à l'encontre d'une harmonisation comptable par directives européennes}

À partir du milieu des années 1990, la Commission s'engage dans une voie remettant totalement en cause l'harmonisation comptable européenne par directives pour l'établissement des comptes consolidés des sociétés faisant appel public à l'épargne.

L'abandon de toute velléité d'harmonisation comptable européenne par directives est clairement annoncé dans une communication de la Commission datée de novembre $1995^{5}$. Ce texte d'une douzaine de pages est structuré autour de quatre points : le contexte actuel de la normalisation comptable, la nécessité de développer une nouvelle approche en matière d'harmonisation comptable, les types de solutions examinées et enfin l'approche proposée. La lecture de cette communication est riche d'enseignements sur la perception que peut avoir la Commission sur la directive en tant que dispositif d'harmonisation comptable au niveau européen. C'est principalement au niveau du contexte et de la nécessité de développer une nouvelle approche que les critiques à l'égard des directives sont les plus virulentes. En effet, la Commission souligne que :

"L'adoption et la transposition des quatrième et septième directives ne se sont réalisées qu'avec difficulté et, depuis lors, aucun nouveau progrès n'a été enregistré au niveau de l'U.E en ce qui concerne l'harmonisation des règles fondamentales de la comptabilité et de l'information financière. Il y a désaccord entre les États membres sur l'utilité de la directive en tant qu'instrument d'harmonisation comptable : certains États membres préféraient une harmonisation internationale plus large et/ou une harmonisation fondée

5 Commission européenne, "L'harmonisation comptable: une nouvelle stratégie au regard de I'harmonisation internationale », COM 95 (508) 
sur des normes plutôt que sur une législation spécifique »(Commission européenne, COM 95 (508), p. 4).

D'emblée c'est l'historique des processus d'élaboration des directives qui est pointé du doigt. C'est un constat d'inefficacité que dresse la Commission tant au niveau de son processus de production de normes, qu'au niveau des structures à même de devoir faire évoluer le corpus de règles comptables. Dès lors que les entreprises publiant des comptes consolidés se financent de plus en plus sur les marchés internationaux, la Commission s'interroge sur la pertinence de poursuivre une réglementation comptable strictement européenne.

Plus loin, nous pouvons lire que « lors de la conférence sur l'avenir de l'harmonisation des normes comptables dans l'Union européenne, organisée par la Commission en 1990, des préférences se sont clairement exprimées : elles étaient hostiles à une réduction du nombre des options offertes par les directives ou à l'adoption de nouveaux textes législatifs dans un avenir proche et favorables à une prise en compte, jugée nécessaire, des efforts d'harmonisation entrepris à un niveau international plus large » (Commission européenne, COM 95 (508), p. 4). En abandonnant les directives comptables, la Commission ne fait que constater l'échec du dispositif et la situation de blocage. Échec relatif car les États membres restent très attachés aux options offertes dans la directive européenne. Options qui permettent dans le meilleur des cas d'obtenir l'accord politique, mais rarement de permettre une réelle harmonisation des états financiers consolidés.

Sans développer davantage, la Commission précise que «les comptes que préparent ces entreprises, conformément à une législation nationale fondée sur les directives comptables, ne sont plus jugés adaptés aux besoins du marché international des capitaux (Commission européenne, COM 95 (508), p. 6). Les critiques à l'égard de la directive se font encore plus cinglantes lorsque l'on peut lire « d'autres problèmes concernent les directives ellesmêmes. Celles-ci autorisent des options multiples et omettent de traiter un certain nombre de questions comptables qui, depuis leur date d'adoption, ont pris une importance croissante. Ces problèmes auxquels il convient d'ajouter les divergences entre États membres sur l'interprétation des principes contenus dans les directives, ont eu des conséquences négatives pour la comparabilité des comptes » (Commission européenne, COM 95 (508), p. 6). La Commission dresse le constat qu'un instrument juridique prévu dans le traité de Rome (1957) n'est plus adapté pour traiter des bouleversements introduits par l'évolution des technologies de l'information, par l'accroissement des échanges économiques et financiers liés à la globalisation. Une fois de plus, le dispositif juridique (la directive) est attaqué dans ses fondements car la souplesse introduite par les options est désormais décriée au motif qu'elle nuit à l'objectif de comparabilité des états financiers. L'incomplétude et la rigidité de l'instrument sont également soulignées. La Commission européenne remet donc totalement en cause la régulation comptable à partir des directives pour la publication des comptes consolidés, ce dispositif lui apparaissant à la fois trop rigide et trop souple...

Enfin, lorsque la Commission présente les solutions envisagées, elle ne manque pas de souligner l'inutilité d'actualiser les directives :

«Une autre solution partielle, portant sur les problèmes internes qui ont été identifiés, consisterait à actualiser les directives comptables en y incluant des solutions techniques aux divers problèmes qu'elles ne traitent pas encore. Il serait toutefois difficile de s'accorder sur le choix des questions susceptibles d'être couvertes par une telle révision. Certains États membres pourraient chercher à renégocier des parties des directives qui ne les satisfont 
pas. La préparation et la négociation d'une révision aussi importante des directives prendraient beaucoup de temps et il est à craindre que, le temps que ces modifications soient finalement adoptées et transposées dans les États membres, de nouveaux problèmes seront déjà apparus ». (Commission européenne, COM 95 (508), p. 8).

Rigidité, obsolescence rapide, crainte que l'édifice fragile de la normalisation comptable ne s'écroule sous les coups de boutoirs d'États membres revanchards amènent la Commission à tourner la page d'une normalisation comptable par directives pour l'établissement des comptes consolidés.

Alors que la directive européenne constituait un élément essentiel du droit communautaire (certes imparfait), pourquoi les critiques de la Commission sont-elles si virulentes? Comment expliquer une telle méfiance à l'égard des États membres dans l'éventualité d'une actualisation des directives?

C'est au travers d'une analyse historique du processus d'élaboration de la Septième directive européenne qu'il nous sera permis de trouver certains éléments de réponse.

\section{Le poids de l'histoire de l'harmonisation comptable européenne}

Vangermeersch (1985), Diggle et Nobes (1994) et Van Hulle et Van der Tas (1995) ont publié des travaux très fouillés sur le processus d'élaboration de la Septième directive européenne. Notre ambition n'est pas ici de relater de nouveau le processus d'élaboration de la Septième directive, mais de comprendre l'origine des critiques à l'égard des directives et ce, une dizaine d'années après son adoption. Cette compréhension passe par une mise en lumière du processus d'élaboration de la directive afin d'identifier les oppositions entre les délégations nationales. Nous ne reprendrons pas l'intégralité du processus mais l'attention se sera focalisée sur la phase de dénouement (action du Coreper) permettant l'obtention de l'accord politique.

\subsection{Le mémorandum du Groupe d'études des experts-comptables de la Communauté économique} européenne (1974)

Pour la Commission européenne, faire appel à un organisme indépendant pour la rédaction d'un rapport était chose courante (Reynaud-Fourton, 1984 ; Tucci, 1985). Sur ce point Vangermeersch (1985) souligne que la Commission accordait une importance non négligeable aux avis émis par le Groupe d'études des experts-comptables de la CEE 6 .

C'est en février 1974 que la Commission des communautés européennes a sollicité le Groupe d'études des experts-comptables de la CEE (GEEC) pour avis sur le projet relatif à la coordination des législations en matière de comptes consolidés. Ce projet était issu des travaux de la commission Elmendorff (1970); laquelle s'est appuyée sur la loi sur les sociétés allemandes de 1965 (Aktiengesetz).

\footnotetext{
${ }^{6}$ Issu de I'Union européenne des experts-comptables économiques et financiers (UEC) mise en place en 1951, le Groupe d'études des experts-comptables de la CEE (GEEC) fut créé en 1961. Composé d'un représentant par État membre et d'un ou plusieurs conseillers techniques, le GEEC rédigeait, entre autres, des études techniques destinées à la Commission des communautés européennes. Pour plus de précisions voir Camfferman et Zeff (2009).
} 
Les commentaires du GEEC sont consignés dans un document de six pages Mémorandum sur les règles à préconiser en matière de consolidation des comptes publiée en 1974. Neuf points sont successivement abordés : la nécessité de disposer de comptes consolidés (1), le besoin de simplicité (2), l'objectif des comptes consolidés (3), la définition du groupe pour les besoins des comptes consolidés (4), l'exclusion de certaines filiales (5), la forme et le contenu des comptes consolidés (6), les règles pour la préparation des comptes consolidés, traitement des résultats provenant des opérations intersociétés/traitement des intérêts minoritaires/comptabilisation des prises de participation et différence de consolidation/conversion des comptes en monnaies étrangères (7), les sociétés associées et « joint-ventures » (8) et enfin la révision des comptes consolidés (9).

D'emblée, le GEEC s'accorde sur le fait que seule la présentation de comptes consolidés peut donner « une image fidèle des actifs, des passifs, de la situation financière et des résultats du groupe ». (GEEC, 1974, p. 2). Il précise également qu'une société devrait établir des comptes consolidés dès lors qu'elle exerce un contrôle sur d'autres sociétés. Le contrôle existe lorsque la société mère :

- "possède, directement ou indirectement, plus de la moitié des actions ordinaires avec un droit de vote de plus de $50 \%$;

- détient par contrat, le pouvoir de nommer la majorité des membres du conseil de direction;

- dispose de la majorité effective dans l'assemblée. »

À l'adresse de la Commission, le GEEC souligne cependant que la directive en projet devrait exiger la présentation de comptes consolidés dans le premier cas de contrôle et se limiter à la permettre dans les deux autres cas. Bien que la Quatrième directive européenne ne soit pas encore adoptée, le GEEC indique que les comptes consolidés devront se conformer à ses prescriptions. Il estime d'ailleurs qu'il est inutile de spécifier des règles détaillées de consolidation dans la directive.

Les joint-ventures sont définies par le Groupe d'études comme des entités économiques créées entre un nombre limité de sociétés pour servir de base à la réalisation de leurs opérations en commun. Prudent sur la manière d'intégrer ces sociétés dans le périmètre de consolidation, il considère que la méthode de l'intégration proportionnelle devrait être permise sans être obligatoire.

Le GEEC considère que compte tenu de la complexité de la consolidation, la directive devrait être la plus générale, se limiter seulement à formuler des règles fondamentales et laisser aux organismes nationaux et internationaux de normalisation le soin de définir des règles précises. Par la suite, ce projet fut soumis au Comité économique et social de la Communauté européenne et au Parlement européen pour avis

\subsection{Les avis du Comité économique et social (1977) et du Parlement de la CEE (1978)}

\subsubsection{L'avis du Comité économique et social du 26 mars 1977}

Dans son ensemble, le Comité économique et social approuve la proposition de directive. Il formule néanmoins le vœu qu'il n'y ait pas de discordances entre cette directive et la norme IAS 3 « Consolidated financial statements » publiée en 1976. D'ailleurs, il invite à une réécriture de certains articles afin que ceux-ci soient conformes aux normes de l'IASC'.

\footnotetext{
7 Journal Officiel des Communautés Européennes, $n^{\circ} \mathrm{C} 75$, du 26 mars 1977, p. 7.
} 
Soucieux d'améliorer l'information financière, le Comité économique et social propose d'introduire un tableau de financement dans les états financiers consolidés. ${ }^{8}$ En même temps, il s'inquiète de la divulgation d'informations jugées confidentielles lorsque la consolidation concerne des petits groupes. L'avis rendu par le Comité économique et social ne remet pas en cause l'économie générale de la proposition de Septième directive européenne. Toutefois, il pointe certains problèmes qu'il serait souhaitable de régler dans la proposition modifiée.

\subsubsection{L'avis du Parlement européen du 10 juillet 1978}

L'accueil réservé par le Parlement européen à la proposition de Septième directive européenne fut très favorable comme le montre l'avis rendu le 10 juillet 1978. Il approuve le recours aux notions d'influence notable et d'influence dominante qui permettent à la directive de s'appliquer aux montages juridiques utilisés pour constituer des groupes. Il juge aussi approprié le critère de direction unique et l'extension de l'obligation de consolidation aux « groupes horizontaux ». De même, il considère comme conforme aux objectifs de la directive, l'inclusion dans le périmètre de consolidation, des sociétés dont le siège social est hors de la Communauté. Le Parlement européen est favorable à ce que les entreprises non significatives puissent être écartées du périmètre de consolidation. Il approuve dans son ensemble les dispositions relatives à l'annexe et les principes généraux d'évaluation.Les deux avis étant rendus, la Commission publia le 12 décembre 1978 une proposition de Septième directive européenne.

\section{3. À la recherche de l'accord politique}

L'objectif visé par la coordination des législations en matière de consolidation était de rendre équivalents et comparables les états financiers consolidés des sociétés européennes. Comme le montre l'examen des débats, de profondes divergences existaient entre les différentes délégations des États membres.

\subsubsection{L'affrontement autour des modèles comptables au sein du Groupe des questions économiques} du Conseil (1979-1981)

La proposition modifiée fut discutée au sein du Conseil par le Groupe des questions économiques (section Établissement et Services) qui réunissait les délégations allemande, italienne, britannique, néerlandaise, belge, luxembourgeoise, danoise, irlandaise et française. Cette dernière comprenait trois représentants issus du ministère de la Justice, du Conseil national de la comptabilité et de la Commission des opérations de bourse. Les réunions du Groupe des questions économiques, qui débutèrent le 26 janvier 1979, se succédèrent au rythme de deux par mois tout au long de l'année 1979. La proposition modifiée de Septième directive européenne est finalement très éloignée de la version initiale (Vangermeersch, 1985 ; Diggle et Nobes, 1994). Les débats du Groupe des questions économiques ont porté sur les points suivants :

- le traitement des entreprises dans lesquelles une société a une participation minoritaire avec pouvoir effectif de contrôle (point $\mathrm{n}^{\circ} 1$ );

- le traitement des entreprises dans lesquelles une société a une participation majoritaire mais n'exerce pas un pouvoir effectif de contrôle (point $n^{\circ} 2$ );

\footnotetext{
${ }^{8}$ Journal Officiel des Communautés Européennes, nC75, du 26 mars 1977, p. 7.
} 
- les sociétés mères qui ne sont pas des sociétés de capitaux (sociétés de personnes, personnes physiques) (point $n^{\circ} 3$ );

- le groupe horizontal (point $n^{\circ} 4$ );

- la sous consolidation (point $\mathrm{n}^{\circ} 5$ );

- les seuils à retenir pour la consolidation (point $\left.n^{\circ} 6\right)$.

Sur les points $n^{\circ} 1$ et $n^{\circ} 2$, les délégations britannique et irlandaise obtinrent des services de la Commission que les critères à retenir pour déterminer les entreprises à consolider soient des critères objectifs tels que :

- la détention par une entreprise de plus de la moitié des droits de vote dans une autre entreprise;

- le pouvoir légal de désigner plus de la moitié des dirigeants de cette entreprise;

- le pouvoir donné à une entreprise d'exercer par contrat avec une autre entreprise le pouvoir légal de contrôle de cette dernière.

Ces entreprises seraient alors incluses dans le périmètre de consolidation et intégrées par la méthode de l'intégration globale. Partiellement en désaccord avec cette conception, la délégation française estimait qu'il fallait aussi intégrer le cas où la société mère disposait d'une participation suffisamment importante pour exercer sur une filiale un contrôle de fait. Dans un premier temps, la Commission avait repris cette suggestion dans des termes assez évasifs : "Sont comprises dans les comptes consolidés les entreprises dans lesquelles une autre entreprise exerce effectivement une influence dominante ».

Cette formulation fut refusée par les délégations britannique et irlandaise en raison du caractère trop subjectif de la notion $\mathrm{d}^{\prime}$ ' « influence dominante ».

Pour sortir de l'impasse, la délégation française proposa le 16 novembre 1979 une rédaction plus explicite des situations de contrôle de fait :

«Rédiger comme suit l'article 6, paragraphe 1, lettre d:

«...d) détient au moins $20 \%$ des droits de vote existant dans une autre entreprise (dite entreprise filiale) à condition :

d'une part que l'exercice effectif de ces droits lui ait permis de désigner à elle seule la majorité (la moitié au moins) des membres en fonction de l'organe d'administration, de direction ou de surveillance de ladite entreprise et de faire adopter par les assemblées générales tenues au cours de l'exercice écoulé les décisions proposées par lesdits organes. D'autre part qu'il existe entre les deux entreprises concernées une unité de direction de caractère durable résultant notamment de l'existence d'un organe commun de direction, ou de la présence des mêmes personnes ou de personnes agissant pour leur compte dans les organes d'administration de direction ou de surveillance des deux entreprises ou de tout autre arrangement $» .9$

La Commission trouva alors la note de la délégation française trop précise mais fut prête à l'accepter si elle était sensiblement allégée.

Le but de la délégation française était de faire en sorte que la Commission fasse figurer dans sa proposition au Conseil, un paragraphe relatif au traitement des entreprises minoritaires avec contrôle effectif, qui soit de nature à rallier la Commission, les délégations allemande et italienne.

\footnotetext{
9 Archives professionnelles privées de Bertrand d'Illiers: Conseil des Communautés européennes: $n^{\circ}$ 10764/79 TRA, Note de transmission de la délégation française, 16 novembre 1979.
} 
Sur la question du statut juridique de la société à la tête du groupe (point $n^{\circ} 3$ ), aucune modification ne fut apportée aux dispositions imposant aux sociétés de personnes et entreprises individuelles de présenter des comptes de groupe dès lors qu'elles sont l'entreprise dominante dans un groupe qui contient au moins une société de capitaux. Cette disposition posait problème car la Septième directive européenne s'inscrivait dans le prolongement de la Quatrième directive qui ne concernait que les sociétés de capitaux. Pour passer outre, les États membres auraient la possibilité d'exempter certaines sociétés de cette obligation en fixant des seuils (total du bilan, chiffre d'affaires, effectif).

Sur la question des groupes horizontaux ${ }^{10}$ (point $\left.n^{\circ} 4\right)$, la Commission estimait que les arguments avancés pour les groupes verticaux étaient aussi valables pour les groupes horizontaux. Cette position n'était pas partagée par toutes les délégations. En effet, elle fut fermement repoussée par les délégations britannique et irlandaise. Elle fut défendue par les délégations des Pays-Bas et de la Belgique. Enfin, les délégations française et allemande y étaient favorables mais souhaitaient que soit introduit un caractère facultatif.

La question de la sous-consolidation (point $n^{\circ} 5$ ) fut aussi très débattue. La Commission estimait que celle-ci était utile. Les délégations française, allemande et britannique firent remarquer l'importance de la charge de travail supplémentaire que constituait cette sous-consolidation. Elles obtinrent de la Commission qu'elle renonce à prévoir une obligation de sous-consolidation généralisée.

Enfin, sur la question des seuils (point $n^{\circ} 6$ ), l'obligation d'établir des comptes consolidés n'était prévue qu'à l'égard des groupes d'une certaine importance. Les seuils proposés par la Commission furent jugés trop bas par les délégations française et allemande. Ces dernières souhaitaient les relever afin que les groupes de petite taille n'aient pas à consolider, le but étant de ne pas imposer la consolidation à des groupes plus petits que les groupes cotés.

Lorsque l'Italie assura la présidence de la Communauté Économique européenne du

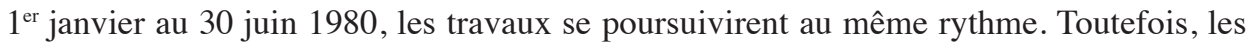
délégations ne parvenaient pas à trouver de compromis. Pour preuve, cet extrait du compte rendu de la réunion des 10 et 11 janvier 1980 du Groupe des questions économiques :

"La délégation française, appuyée par les représentants de la Commission, a souligné l'importance que revêt pour elle le maintien d'un dispositif visant la consolidation des participations minoritaires en cas de contrôle effectif, étend entendu que, pour les participations majoritaires, le Groupe des questions économiques a fait des concessions importantes à la délégation irlandaise et à celle du Royaume-Uni en acceptant le concept de pouvoir légal de contrôle. Une solution facultative pour une question si significative ne tiendrait pas compte des buts de la directive $» .^{.1}$

Lorsque le $1^{\text {er }}$ juillet 1980, le Luxembourg commença à exercer la présidence de la Communauté Économique Européenne, son enthousiasme modéré vis-à-vis de la Septième directive faisait craindre le pire aux délégations qui souhaitaient arriver rapidement à un accord. Ces craintes étaient totalement justifiées puisque le rythme des réunions passa de deux à une par mois. Cette mesure suscita la désapprobation des délégations allemande, française, italienne, néerlandaise, belge, danoise et des représentants de la Commission.

\footnotetext{
${ }^{10}$ Le groupe horizontal (ou consortium) existe dès lors que des entreprises se rassemblent pour former un groupe dont la caractéristique fondamentale est que les entreprises concernées ont une direction unique et centralisée. Cette direction est le fait conjoint de toutes les entreprises concernées, mais pas d'une entreprise dominante. Les entreprises chargées d'assurer la direction centralisée et unique travaillent sur un pied d'égalité, il n'y a pas de relation de dépendance dans ce type de groupe.

11 Archives professionnelles privées de Bertrand d'Illiers: Conseil des Communautés européennes: $n^{\circ}$ 4402/80, 23 janvier 1980, p. 4.
} 
Malgré ces protestations, le rythme fut maintenu à une réunion mensuelle. Fin 1980, sur les vingt-sept articles que comprenait la proposition modifiée, seulement 11 articles avaient été examinés. Les points de désaccord demeuraient encore nombreux.

Le Groupe des questions économiques ayant achevé quatre lectures des articles relatifs aux principes généraux, faisait le constat qu'il ne lui était plus possible de progresser à son niveau. Il décida donc de soumettre un rapport intermédiaire au Comité des représentants permanents (COREPER) afin de sortir de l'impasse.

\subsubsection{L'action décisive du COREPER (1982-1983)}

Le Comité des représentants permanents (COREPER), formé des représentants permanents des États membres et d'ambassadeurs, était chargé d'assister le Conseil en préparant les dossiers sur lesquels celui-ci devait prendre position. Il se consacrait à une phase de «pré-négociation » afin de faciliter les prises de décisions du Conseil. Les représentants permanents organisaient le dialogue entre les États et la Commission.

\section{Le programme de travail du COREPER}

Au sein du COREPER, c'est le Groupe ad hoc des conseillers des représentations permanentes qui examina le rapport intermédiaire. Les points encore en suspens portaient sur :

- La consolidation verticale en cas de participation majoritaire (art 6 §1a, §1b, §1c) : Ces dispositions prévoyaient l'obligation d'établir des comptes consolidés par toute entreprise disposant du contrôle juridique sur une autre entreprise en raison : de la possession de la majorité des droits de vote; ou du droit de nommer ou de révoquer la majorité des membres des organes d'administration, de direction ou de surveillance ou du droit d'exercer une influence dominante en vertu d'un contrat ou d'une disposition statutaire. Sur ce point, à l'exception de la délégation luxembourgeoise, toutes les délégations étaient d'accord.

- La forme juridique des entreprises comprises dans la consolidation (art 6 §4 et §6) : le paragraphe 4 de l'article 6 prévoyait : "l'établissement de comptes consolidés si au moins l'entreprise mère ou une de ses filiales était organisée dans la forme de société capitaux au sens de la Quatrième directive ». Les délégations française, irlandaise et britannique souhaitaient que cette obligation ne s'adresse qu'aux sociétés mères ayant la forme de sociétés de capitaux. Plus restrictive dans son approche, la délégation luxembourgeoise désirait que ne soient incluses dans le périmètre de consolidation que des sociétés de capitaux. À l'inverse, la délégation allemande demandait que la consolidation soit étendue à toute entreprise à responsabilité limitée ou ayant une forme comparable. Sur ce dernier point, les autres délégations faisaient remarquer que cette position soulevait le problème de l'application de la Septième directive à des sociétés non concernées par la Quatrième directive.

- L'exemption de consolider des ensembles d'entreprises de taille limitée (art 6 §7) : Cette disposition prévoyait une exemption totale de consolidation, si l'ensemble des entreprises à consolider ne dépassait pas deux des trois limites suivantes :

- total du bilan : 1 million d'écus;

- chiffre d'affaires : 2 millions d'écus;

- nombre de salariés : 50.

Les divergences portaient tant sur le mode de calcul que sur le niveau des seuils. Ces derniers, qui correspondaient à ceux définis dans la Quatrième directive, ne satisfaisaient 
pas les délégations française, belge et irlandaise. Ces dernières souhaitaient 4 millions d'écus pour le total du bilan, 8 millions d'écus pour le chiffre d'affaires et 250 pour le nombre de salariés. Les délégations allemande, italienne, néerlandaise et luxembourgeoise réclamaient un relèvement encore plus important. Les délégations britannique et danoise s'opposaient à tout relèvement...

Pour les groupes horizontaux réels, les délégations allemande, belge, italienne et néerlandaise ainsi que les représentants de la Commission étaient favorables au caractère obligatoire de la consolidation horizontale, alors que les délégations danoise, irlandaise, luxembourgeoise et britannique faisaient part de leurs réserves. De son coté, la délégation française était prête à se rallier à la majorité.

\section{Les résultats du COREPER}

Le Groupe ad hoc des conseillers des représentations permanentes se réunit à huit reprises, entre le $1^{\text {er }}$ mars et le 27 avril 1982, pour examiner les orientations de compromis du Groupe des questions économiques. En juin 1982, il n'avait réussi à trouver un compromis que sur des points mineurs. Il estima alors qu'il n'était plus possible de faire de progrès à son niveau et appela le Conseil à se prononcer sur les points restés en suspens ${ }^{12}$. Ces points concernaient :

- la demande de la délégation luxembourgeoise de prévoir, comme condition supplémentaire de l'obligation de consolidation, l'exercice effectif du contrôle vis-à-vis des filiales ;

- le principe de l'inclusion «des sociétés holdings de type luxembourgeois ${ }^{13}{ }^{»}$ dans la directive;

- le maintien de l'article 6 §1d relatif à la consolidation en cas de participations minoritaires ;

- la question de savoir si la faculté d'exemption pour les États membres peut être basée sur le seul fait que l'entreprise mère n'est pas une société de capitaux ;

- les seuils à retenir;

- les exemptions des ensembles d'entreprises de taille limitée.

N'ayant pu parvenir à un accord, le Conseil saisit de nouveau le COREPER pour qu'il trouve un compromis sur la proposition modifiée de Septième directive.

Dix réunions supplémentaires du COREPER furent nécessaires entre décembre 1982 et mars 1983 pour aboutir à un accord communautaire.

L'accord général fut enfin obtenu lors de la réunion du COREPER le 4 mai 1983. Le texte fut transmis dans sa version définitive au Conseil pour accord politique le 16 mai 1983. Le 13 juin 1983, la Septième directive était enfin adoptée par le Conseil des communautés européennes soit presque de dix ans après le projet de texte initial.

\footnotetext{
${ }^{12}$ Archives professionnelles privées de Bertrand d'Illiers : Rapport du Comité des conseillers des représentations permanentes au Conseil, le 3 juin 1982 (7494/82 DRS 33).

${ }^{13}$ Les « sociétés holdings de type luxembourgeois » sont des sociétés de participation financière au sens de l'article 5 paragraphe 3 de la Quatrième directive européenne. Selon lequel : «Par sociétés de participation financière au sens de la présente directive, on entend exclusivement les sociétés dont l'objet unique est la prise de participations dans d'autres entreprises ainsi que la gestion et la mise en valeur de ces participations sans que ces sociétés s'immiscent directement ou indirectement dans la gestion de ces entreprises, sans préjudice des droits que les sociétés de participation financière détiennent en leur qualité d'actionnaires ou d'associés. Le respect des limites imposées aux activités de ces sociétés doit pouvoir être contrôlé par une autorité judiciaire ou administrative. »(Art $5 \S 3$ de la Quatrième directive européenne).
} 


\section{Discussion}

La stratégie de l'Union européenne en matière d'harmonisation comptable a déjà été largement commentée. Notre objectif n'est pas de revenir sur le choix de la Commission européenne. Il est de comprendre l'origine des critiques formulées à l'égard de la Septième directive comme mode d'harmonisation comptable dans un environnement économique et financier devenu totalement mondialisé.

\subsection{La lourdeur du processus de coordination du droit des sociétés à partir des directives}

Le désaveu à l'égard des directives européennes trouve son origine dans la lourdeur du processus retracé dans la section 2 ci-dessus. L'extrême lenteur du processus d'harmonisation par directives est un point crucial que l'on retrouve à plusieurs reprises dans le texte de la communication de la Commission et qui explique, en partie seulement, le choix de la Commission de se rapprocher de l'IASC. Nous l'avons vu, la négociation de la directive a pris près d'une dizaine d'années; auxquelles il faut ajouter le délai nécessaire de transposition de la directive dans les droits nationaux des États membres. La transposition fut assez rapide pour la France car elle est intervenue seulement 18 mois après l'adoption de la directive. En revanche, elle fut beaucoup plus tardive pour des pays tels que le RoyaumeUni (78 mois) et l'Irlande (114 mois) après son adoption en juin 1983 (Alexander et Nobes, 1994 ; Hopwood, 1994 ; Môme, 1995).

La lenteur inhérente au mode de fonctionnement des institutions communautaires explique aussi ce renoncement. En effet, pas moins de six d'entre elles sont intervenues dans le processus ${ }^{14}$. C'est cette lenteur et cette lourdeur qui sont dénoncées avec force dans la communication de la Commission annonçant sa nouvelle stratégie en matière d'harmonisation comptable.

\subsection{Les options : condition sine qua none de l'accord politique au détriment d'une véritable coordination des législations}

La Septième directive traite d'une réalité économique (les groupes) commune à tous les États membres mais pour laquelle (à l'exception notoire de l'Allemagne) aucun n'a encore reconnu d'existence juridique. En conséquence, des débats interminables ont porté sur le champ d'application de la consolidation et sur le périmètre de consolidation. Sur ce point, et sur bien d'autres, les divergences entre les délégations française et britannique traduisaient les approches fondamentalement différentes de la notion de groupe. Diggle et Nobes (1994) ont mis en évidence le glissement progressif d'une conception ancrée dans le droit allemand vers une conception davantage orientée vers un modèle anglo-saxon de la comptabilité.

La difficulté d'aboutir à un accord politique et l'introduction des options dans le texte final sont aussi clairement dénoncées. Si ces options ont permis l'adoption de la directive le 13 juin 1983, la présence massive de ces dernières a été largement critiquée (Van Hulle,

\footnotetext{
14 La Commission des communautés européennes, le Conseil, le Comité économique et social, le Parlement européen, le Groupe des questions économiques (établissement et services) du Conseil et enfin le Groupe ad hoc des conseillers des représentations permanentes du COREPER
} 
1992 ; Thorell and Whittington, 1994; Van Hulle, 1996; Haller, 2002). Sur ce point, on peut s'interroger sur les motivations de la Commission à critiquer le recours aux options. En effet, c'est l'objectif même de la directive que de fournir un texte de portée générale. C'est ensuite aux États membres et aux organismes nationaux de normalisation de préciser certains points techniques (Hoarau, 1995).

\subsection{Un niveau très hétérogène de la pratique de la consolidation des comptes dans les États membres}

Une des difficultés d'obtention d'un accord rapide s'explique notamment par une maitrise imparfaite des enjeux de la consolidation et une forte différence des niveaux de pratique entre États membres. À titre d'exemple, la pratique de consolidation des comptes est apparue au Royaume-Uni dès les années 1910 (Edwards et Webb, 1984). Elle devient obligatoire avec le Company Act de 1948. Au moment où la directive est négociée, la consolidation des comptes est une pratique courante des sociétés anglaises. En revanche, pour certains pays comme la Grèce, la pratique de la consolidation est encore peu développée (Van Hulle et Van der Tas, 1995).

Dans une position intermédiaire, la France dispose depuis 1968 d'une réglementation comptable sur les comptes consolidés. Elle fut mise au point avec quelques grandes compagnies françaises de dimension internationale sous l'égide du Conseil National de la Comptabilité. Même si la Commission des opérations de bourse dénonce la piètre qualité des états financiers consolidés publiés par les sociétés françaises en raison d'un vagabondage comptable, il existe en France une connaissance des enjeux et des pratiques de la consolidation (Bensadon, 2013).

$\mathrm{Si}$, sur le principe, existait une volonté de rendre comparables et équivalents les comptes consolidés, dans la pratique les intérêts nationaux défendus par les délégations venaient vite prendre le pas, certaines délégations (française et allemande) venant même à douter du succès des négociations.

Ainsi, la lourdeur du processus d'harmonisation comptable par directives, l'hétérogénéité du niveau de pratique de la consolidation des comptes dans les États membres et le recours aux options peuvent expliquer les critiques que formule la Commission à l'égard des directives et justifier son choix en faveur d'un rapprochement vers l'IASC de l'époque. Cependant, un autre élément essentiel explique pourquoi la Commission se détourne d'un processus de régulation comptable par directives pour les comptes de groupe :l'influence de l'OICV.

\subsection{L'influence de l'Organisation Internationale des Commissions de Valeurs (OICV)}

Dans cette bataille autour de l'élaboration du dispositif de normalisation comptable, le rôle de l'Organisation internationale des commissions de valeurs (OICV) est considérable. L'OICV qui compte 114 membres (dont 70 régulateurs boursiers nationaux) fut créé en 1965 (Perier, 1995).

L'objectif de l'OICV en matière comptable fut la constitution d'un référentiel de normes utilisables par les émetteurs souhaitant lever des capitaux sur d'autres marchés que celui de leur pays d'origine. Juillet 1995 marque un tournant dans le processus de convergence 
des normes comptables. En effet, l'OICV a tenu à Paris sa vingtième conférence annuelle, à l'issue de laquelle l'OICV et l'IASC ont arrêté une liste de domaines comptables devant faire l'objet de nouvelles normes ou d'une révision. Cet adoubement de l'IASC par l'OICV allait sonner le glas de l'harmonisation comptable européenne par directives pour les sociétés devant publier des comptes consolidés. Au même moment, Mario Monti, alors commissaire européen, apportait son soutien aux efforts de l'IASC en vue de développer un corps de normes unique et affirmait que l'Europe ne chercherait pas à mettre en place un comité de réglementation comptable propre.

\section{Conclusion}

L'idée d'une harmonisation comptable européenne a vu le jour quelques années après la signature du traité de Rome. Les réflexions autour de la forme qu'elle devait prendre ont privilégié la coordination des législations nationales. Dans un contexte encore peu marqué par la globalisation financière, le temps long de la négociation entre un nombre limité d'États membres a constitué la voie normale de l'harmonisation comptable.

En revanche, dans un contexte totalement bouleversé par l'émergence des nouvelles technologies de l'information et de la communication, par les innovations financières complexes et par la financiarisation, le processus d'harmonisation comptable par les directives européennes atteignait ses limites. Face à la montée en puissance prévisible des normes IAS/IFRS (Accord de l'OICV), la Commission européenne allait changer radicalement de stratégie en matière d'harmonisation comptable.

Dans un tel contexte, l'idée d'une harmonisation comptable par directives est rejetée avec force. Il y a certes, avec l'internationalisation des marchés financiers, un courant favorable à un rapprochement vers l'IASC. Cependant, le poids de l'histoire, dont on retrouve les traces dans le texte de la communication de la Commission européenne, permet également d'expliquer ce choix. En renonçant aux directives européennes pour réglementer l'élaboration et l'établissement des comptes consolidés des sociétés faisant appel public à l'épargne, la Commission européenne faisait-elle le choix d'abandonner toute ambition d'harmonisation comptable régionale?

L'adoption en juin 2013 de la directive comptable unique nous apporte un élément de réponse. Amnésie, schizophrénie, inconstance des institutions européennes face à la question de la régulation comptable européenne? Il n'en est rien. La réglementation de l'établissement des comptes sociaux porte sur d'autres enjeux (notamment fiscaux) sur lesquels les États entendent conserver une totale souveraineté.

Afin d'élargir notre analyse, il serait pertinent de compléter cette recherche par une étude des processus de lobbying au sein des institutions européennes de régulation comptable (Sutton, 1984 ; Joos et Lang, 1994 ; Colasse, 2004) mais aussi de mettre en évidence l'institutionnalisation d'une mémoire organisationnelle (Walsh et Ungson, 1991; Girod, 1995) au sein de ces mêmes institutions. 


\section{Bibliographie}

Alexander. D. and Nobes. C., 1994. A European Introduction to Financial Accounting. Prentice-Hall, London

Azan W., 2002. Évolution des systèmes comptables, contrôle externe et réseaux de culture : Kontrag et NRE. Comptabilité - Contrôle - Audit 8 (2), 29-50.

Bensadon D., 2013. À l'origine de la convergence des normes comptables françaises vers le modèle anglo-saxon In Levant Y., Zimnovitch H. et Sandu R. (dir), Mélanges en l’honneur du professeur Yannick Lemarchand. L'harmattan, Paris, 192-217.

Cailluet L. et Lemarchand Y., 2013. L'école d'Orvault? In Cailluet L., Lemarchand Y. et Chessel M.-E. (dir.) Histoire et sciences de gestion, Paris, 1-15

Camfferman K. and Zeff S.A., 2009. The Formation and early Years of the Union européenne des experts Comptables Économiques et Financiers (UEC), 1951-63: or how the Dutch tried to bring down the UEC. Accounting, Business and Financial History 19 (3), 215-257.

Capron M. and Chiapello E., 2005. Les transformations institutionnelles : l'Europe recule, l'IASB s'impose In Capron M. (dir) Les normes comptables internationales, instruments du capitalisme financier, La Découverte, Paris, 49-89.

Chambost I. and Klee L., 2009. La régulation comptable européenne : de l'articulation de l'expertise et du politique. $30^{\mathrm{e}}$ congrès de 1'Association Francophone de Comptabilité, Strasbourg.

Chiappelo E. and Medjad K., 2007. Une privatisation inédite de la norme : le cas de la politique comptable européenne. Sociologie du travail 49, 46-64.

Chiappelo E., 2005. Les normes comptables comme institution du capitalisme : Une analyse du passage aux IFRS en Europe à partir de 2005. Sociologie du travail 47, 362-382.

Colasse B., 2004. Normes comptables : l’Union européenne sous influence ? Sociétal 46, 4 e trimestre.

Colasse B., 2005. La régulation comptable entre public et privé In M. Capron (dir) Les normes comptables internationales, instruments du capitalisme financier, La Découverte Paris, 27-48.

Colasse B., 2009. Harmonisation comptable internationale In B. Colasse (dir) Encyclopédie de ComptabilitéContrôle de gestion et Audit, Economica, Paris, 877-890.

Delvaille P., 2001. L'harmonisation comptable européenne en droit et en pratique. Doctorat en sciences de gestion, Paris, université Paris-Dauphine.

Diggle G. et Nobes C., 1994. European Rule-making in Accounting: The Seventh Directive as a Case Study. Accounting and Business Research 24 (96), 319-333.

Edwards J.R. and Webb. K., 1984. The development of group accounting in the United Kingdom to 1933. The Accounting Historians Journal 11 (1), 31-57.

Frison-Roche M-A., 2011. L'ancrage de la comptabilité dans le droit civil et ses conséquences dans les concepts sous-jacents des normes comptables, Actes des II États généraux de la recherche comptable, Autorité des normes comptables, 33-42.

Girod M., 1995. La mémoire organisationnelle. Revue Française de Gestion 105, 30-42.

Haller A., 2002. Financial Accounting Developments in the European Union: past Events and Future Prospects. European Accounting Review 11 (4), 153-190.

Hoarau C., 1995. International Accounting Harmonization - American Hegemony or Mutual Recognition with Benchmarks. European Accounting Review 4 (2), 217-233.

Hopwood A. G., 1994. Some Reflexions on The harmonization of accounting within the E.U. The European Accounting Review 3 (2), 241-253.

Joos P. and Lang M., 1994. The effects of accounting diversity: Evidence from the European Union. Journal of Accounting Research 32 (supplement), 141-168

Klee L., Chambost I. et Boisselier P., 2012. Tribulations de la régulation comptable européenne : petite histoire d'une construction institutionnelle In C. Simon, C. Hoarau et JL Malo (dir) Comptabilité, contrôle et société «mélanges en l'honneur du professeur Alain Burlaud », Paris, Foucher, 111-129. 
Môme P.-F., 1995. Les comptes consolidés en Europe; émergence du modèle européen de la consolidation. Problématique et mise en cuvre dans le cadre de l'union européenne. Doctorat en sciences de gestion, Nice, université de Nice.

Périer F., 1995. L’Organisation Internationale des Commissions de Valeurs (OICV) et le rôle des régulateurs boursiers dans l'internationalisation des marchés. Revue d'économie financière 33, 123-133.

Reynaud-Fourton C., 1984. La coordination communautaire du droit des sociétés en matière d'information financière: comptes annuels et comptes consolidés (Quatrième et Septième directive). Doctorat en droit, Paris, université Paris I Panthéon-Sorbonne.

Raybaud-Turrillo B., 2001. Les processus de normalisation comptable : un exemple de droit postmoderne. Revue international de droit économique 15 (1), 9-40.

Richard. J, Bensadon D. et Collette C., 2014. Comptabilité financière : Normes IFRS versus normes françaises $10^{\mathrm{e}}$ édition. Dunod, Paris.

Sutton T.G., 1984. Lobbying of accounting standard-setting bodies in the U.K. and the U.S.A.: A Downsian analysis. Accounting, Organizations and Society 9 (1), 81-95

Thorell P. and Whittington G., 1994. The harmonization of accounting within EU - problems, perspectives and strategies. The European Accounting Review 3 (2), 215-239.

Tucci M., 1985. Perspectives introduites par la 7e directive européenne en matière d'information économique et financière sur les groupes d'entreprises. Thèse de $3^{\mathrm{e}}$ cycle en sciences de gestion. Grenoble : université de Grenoble II.

Van Hulle K., 1992. Harmonization of Accounting Standards: a view from the European Community. European Accounting Review 1 (1), 161-172.

Van Hulle K. and Van der Tas L., 1995. "European Accounting Regulation" In Ordelheide and KPMG, Transnational Accounting, Macmillan Press, London.

Van Hulle K., 1996. L'harmonisation comptable européenne : une nouvelle stratégie au regard de l'harmonisation internationale. Revue Française de Comptabilité 275, 29-38.

Vangermeersch R., 1985. The Route of the Seventh Directive of EEC on Consolidated Accounts - Slow, Steady, Studied, and Sucessful. The International Journal of Accounting 20 (2), 103-118.

Walsh J.P., Ungson G., 1991. Organizational memory. Academy of Management Review 16 (1), 57-91.

Walton P., 2003. La normalisation comptable internationale : origines, pratiques et enjeux, Revue française de gestion 147, 21-32.

Zimnovitch H., 2013. Essai sur un rapport critique et fécond entre histoire et gestion In Cailluet L. Lemarchand Y. et Chessel M.-E. (dir.), Histoire et sciences de gestion, Paris, 87-113.

\section{Sources imprimées}

Communication de la Commission au Conseil et au Parlement européen Com (95) 508: l'harmonisation comptable : une nouvelle stratégie au regard de l'harmonisation internationale.

Communication de la Commission au Conseil et au Parlement européen : Com (00) 359 : Stratégie de l'UE en matière d'information financière : la marche à suivre.

Groupe d'études des experts-comptables de la CEE (GEEC), 1974. Mémorandum sur les règles à préconiser en matière de consolidation des comptes.

Journal Officiel des Communautés européennes, 1976. Avis sur une proposition d'une septième directive sur la base de l'article 54 paragraphe 3 sous g) du traité CEE concernant les comptes du groupe, Commission, le 2 juin $1976, \mathrm{n}^{\circ} \mathrm{C} / 121$.

Journal Officiel des Communautés européennes, 1977. Avis sur une proposition d'une septième directive sur la base de l'article 54 paragraphe 3 sous g) du traité CEE concernant les comptes du groupe, Comité Économique et Social, le 26 mars $1977, \mathrm{n}^{\circ} \mathrm{C} / 75$.

Journal Officiel des Communautés européennes, 1979. Avis sur une proposition d'une septième directive sur la base de l'article 54 paragraphe 3 sous g) du traité CEE concernant les comptes du groupe, Commission, le 17 janvier $1979, \mathrm{n}^{\circ} \mathrm{C} / 142$. 